\title{
Uniform asymptotic stability implies exponential stability for nonautonomous half-linear differential systems
}

Masakazu Onitsuka* and Tomomi Soeda

"Correspondence:
onitsuka@xmath.ous.ac.jp
Department of Applied
Mathematics, Okayama University
of Science, Okayama, 700-0005,
Japan

"Correspondence:

Department of Applied

Mathematics, Okayama University Japan

\begin{abstract}
The present paper is considered a two-dimensional half-linear differential system: $x^{\prime}=a_{11}(t) x+a_{12}(t) \phi_{p^{*}}(y), y^{\prime}=a_{21}(t) \phi_{p}(x)+a_{22}(t) y$, where all time-varying coefficients are continuous; $p$ and $p^{*}$ are positive numbers satisfying $1 / p+1 / p^{*}=1$; and $\phi_{q}(z)=|z|^{q-2} z$ for $q=p$ or $q=p^{*}$. In the special case, the half-linear system becomes the linear system $\mathbf{x}^{\prime}=A(t) \mathbf{x}$ where $A(t)$ is a $2 \times 2$ continuous matrix and $\mathbf{x}$ is a two-dimensional vector. It is well known that the zero solution of the linear system is uniformly asymptotically stable if and only if it is exponentially stable. However, in general, uniform asymptotic stability is not equivalent to exponential stability in the case of nonlinear systems. The aim of this paper is to clarify that uniform asymptotic stability is equivalent to exponential stability for the half-linear differential system. Moreover, it is also clarified that exponential stability, global uniform asymptotic stability, and global exponential stability are equivalent for the half-linear differential system. Finally, the converse theorems on exponential stability which guarantee the existence of a strict Lyapunov function are presented.
\end{abstract}

MSC: 34D05; 34D20; 34D23; 37C75; 93D05; 93D20; 93D30

Keywords: uniform asymptotic stability; exponential stability; half-linear differential system; Lyapunov function; converse theorem

\section{Introduction}

We consider a system of differential equations of the form

$$
\begin{aligned}
& x^{\prime}=a_{11}(t) x+a_{12}(t) \phi_{p^{*}}(y), \\
& y^{\prime}=a_{21}(t) \phi_{p}(x)+a_{22}(t) y,
\end{aligned}
$$

where the prime denotes $d / d t$; the coefficients $a_{11}(t), a_{12}(t), a_{21}(t)$, and $a_{22}(t)$ are continuous on $I=[0, \infty)$; the numbers $p$ and $p^{*}$ are positive and satisfy

$$
\frac{1}{p}+\frac{1}{p^{*}}=1
$$

the real-valued function $\phi_{q}(z)$ is defined by

$$
\phi_{q}(z)=\left\{\begin{array}{ll}
|z|^{q-2} z & \text { if } z \neq 0, \\
0 & \text { if } z=0,
\end{array} \quad z \in \mathbb{R}\right.
$$

(c) 2015 Onitsuka and Soeda; licensee Springer. This article is distributed under the terms of the Creative Commons Attribution 4.0 International License (http://creativecommons.org/licenses/by/4.0/), which permits unrestricted use, distribution, and reproduction in any medium, provided you give appropriate credit to the original author(s) and the source, provide a link to the Creative Commons license, and indicate if changes were made. 
with $q=p$ or $q=p^{*}$. Note that $\phi_{p^{*}}$ is the inverse function of $\phi_{p}$, and the numbers $p$ and $p^{*}$ are naturally greater than 1 . Hence, we also note that the right-hand side of (1.1) does not satisfy Lipschitz condition at the origin since the function $\phi_{p}$ satisfies

$$
\lim _{z \rightarrow 0} \frac{d}{d z} \phi_{p}(z)=\lim _{z \rightarrow 0}(p-1)|z|^{p-2}=\infty
$$

if $1<p<2$, and the function $\phi_{p^{*}}$ satisfies

$$
\lim _{z \rightarrow 0} \frac{d}{d z} \phi_{p^{*}}(z)=\lim _{z \rightarrow 0}\left(p^{*}-1\right)|z|^{p^{*}-2}=\infty
$$

if $p>2$. Since $\phi_{p}(0)=0=\phi_{p^{*}}(0)$, system (1.1) has the zero solution $(x(t), y(t)) \equiv(0,0)$. The type of system (1.1) appeared in [1-9]. Let $u=\exp \left(-\int a_{11}(t) d t\right) x$ and $v=\exp \left(-\int a_{22}(t) d t\right) y$. Then we can transform system (1.1) into the simpler system

$$
u^{\prime}=a(t) \phi_{p^{*}}(v), \quad v^{\prime}=b(t) \phi_{p}(u)
$$

where

$$
a(t)=a_{12}(t) \exp \left(\int\left\{\left(p^{*}-1\right) a_{22}(t)-a_{11}(t)\right\} d t\right)
$$

and

$$
b(t)=a_{21}(t) \exp \left(\int\left\{(p-1) a_{11}(t)-a_{22}(t)\right\} d t\right) .
$$

The problem of the global existence and uniqueness of solutions of this type of system are treated in $[1,2,6]$. However, keep in mind that we do not require the uniqueness of solutions of (1.1) throughout this paper.

In the special case that $a_{11}(t) \equiv 0$ and $a_{12}(t) \equiv 1,(1.1)$ is transformed into the equation

$$
\left(\phi_{p}\left(x^{\prime}\right)\right)^{\prime}-a_{22}(t) \phi_{p}\left(x^{\prime}\right)-a_{21}(t) \phi_{p}(x)=0 .
$$

If $x(t)$ is a solution of (1.2), then $c x(t)$ is also a solution of (1.2) for any $c \in \mathbb{R}$; that is, the solution space of (1.2) is homogeneous. However, even if $x_{1}(t)$ and $x_{2}(t)$ are two solutions of (1.2), the function $x_{1}(t)+x_{2}(t)$ is not always a solution of (1.2); that is, the solution space of (1.2) is not additive. For this reason, this equation is often called 'half-linear'. For example, half-linear differential equation or half-linear differential system can be found in [1-19] and the references cited therein. Furthermore, we can confirm that the global existence and uniqueness of solutions of (1.2) are guaranteed for the initial value problem (see $[1,2]$ ). To study half-linear differential equations is important in the field of difference equations, dynamic equations on time scales, partial differential equations and various functional equations, because the method of differential equation might be able to use for them. The reader may refer to [20-24] for example. 
In the other special case, $p=2$, (1.1) becomes the two-dimensional linear differential system

$$
\begin{aligned}
& x^{\prime}=a_{11}(t) x+a_{12}(t) y, \\
& y^{\prime}=a_{21}(t) x+a_{22}(t) y .
\end{aligned}
$$

We now consider more general linear differential systems of the form

$$
\mathbf{x}^{\prime}=A(t) \mathbf{x}
$$

with an $n \times n$ continuous matrix $A(t)$. It is well known that the zero solution of (1.3) is uniformly asymptotically stable if and only if it is exponentially stable (see Section 2 about the precise definitions of uniform asymptotic stability and exponential stability). To be precise, the following theorem holds (for the proof, see ([25],pp.43-44), ([26], p.85), ([27], pp.499-500) and ([28], pp.29-30)).

Theorem A If the zero solution of (1.3) is uniformly asymptotically stable, then it is exponentially stable.

In general, uniform asymptotic stability is not equivalent to exponential stability in the case of nonlinear systems. Actually, we consider the scalar equation $x^{\prime}=-x^{3}$ (see ([26], p.85) and ([29], p.49)). Clearly, this equation has the unique zero solution $x\left(t ; t_{0}, 0\right) \equiv 0$. In the case that $x_{0} \neq 0$, the solution of this equation passing through a point $x_{0} \in \mathbb{R}$ at $t_{0} \in I$ is given by

$$
x(t)=\frac{x_{0}}{\sqrt{1+2 x_{0}^{2}\left(t-t_{0}\right)}} .
$$

It is known that the zero solution of this equation is uniformly asymptotically stable. However, it is not exponentially stable. In fact, we see

$$
|x(t)| e^{\Lambda\left(t-t_{0}\right)}=\frac{x_{0} e^{\Lambda\left(t-t_{0}\right)}}{\sqrt{1+2 x_{0}^{2}\left(t-t_{0}\right)}} \rightarrow \infty \quad \text { as } t \rightarrow \infty
$$

for any $\Lambda>0$. It is clear that the solution $x(t)$ does not converge to the zero solution exponentially; that is, the zero solution is not exponentially stable. Here, the first question of this paper arises. Will uniform asymptotic stability guarantee exponential stability, even if the half-linear differential system (1.1) is nonlinear? We now give the answer to this question.

Theorem 1.1 If the zero solution of (1.1) is uniformly asymptotically stable, then it is exponentially stable.

Uniform asymptotic stability and exponential stability are of utmost importance for control theory. For example, we can assert that these stabilities guarantee the existence of a Lyapunov function which has good characteristics. Such results is called 'converse theorems' on stability. The converse theorems are important in studying the properties of 
solutions of perturbed systems. We will consider the general nonlinear system

$$
\mathbf{x}^{\prime}=\mathbf{f}(t, \mathbf{x})
$$

where $\mathbf{f}(t, \mathbf{x})$ is continuous on $I \times \mathbb{R}^{n}$ and satisfies $\mathbf{f}(t, \mathbf{0})=\mathbf{0}$. We define the set $S_{\alpha}=\{\mathbf{x} \in$ $\left.\mathbb{R}^{n}:\|\mathbf{x}\|<\alpha\right\}$ for $\alpha>0$. Let $\mathbf{x}\left(t ; t_{0}, \mathbf{x}_{0}\right)$ be a solution of (1.4) passing through a point $\mathbf{x}_{0} \in \mathbb{R}^{n}$ at a time $t_{0} \in I$. It is well known that if we suppose that $\mathbf{f}(t, \mathbf{x})$ satisfies locally Lipschitz condition with respect to $\mathbf{x}$ and if the zero solution of (1.4) is uniformly asymptotically stable, then there exists a strict Lyapunov function (or strong Lyapunov function) $V(t, \mathbf{x})$; that is, the scalar function $V(t, \mathbf{x})$ defined on $I \times S_{\alpha}$, where $\alpha$ is a suitable constant, which satisfies the following conditions:

(i) $a(\|\mathbf{x}\|) \leq V(t, \mathbf{x}) \leq b(\|\mathbf{x}\|)$;

(ii) $\dot{V}_{(1.4)}(t, \mathbf{x}) \leq-c(\|\mathbf{x}\|)$,

where $a, b$, and $c$ are continuous increasing and positive definite functions and the function $\dot{V}_{(1.4)}(t, \mathbf{x})$ is defined by

$$
\dot{V}_{(1.4)}(t, \mathbf{x})=\limsup _{h \rightarrow 0+} \frac{V(t+h, \mathbf{x}(t+h ; t, \mathbf{x}))-V(t, \mathbf{x})}{h}
$$

(see [25-36]).

Furthermore, global exponential stability guarantees the existence of better Lyapunov functions in the sense that it can be estimated by exact functions. The definition of global exponential stability is as follows. Let $\|\mathbf{x}\|$ be the Euclidean norm. The zero solution is said to be globally exponentially stable (or globally exponentially asymptotically stable or exponentially asymptotically stable in the large) if there exists a $\lambda>0$ and, for any $\alpha>0$, there exists a $\beta(\alpha)>0$ such that $t_{0} \in I$ and $\left\|\mathbf{x}_{0}\right\|<\alpha$ imply $\left\|\mathbf{x}\left(t ; t_{0}, \mathbf{x}_{0}\right)\right\| \leq \beta(\alpha) e^{-\lambda\left(t-t_{0}\right)}\left\|\mathbf{x}_{0}\right\|$ for all $t \geq t_{0}$. For example, we can refer to the books $[28-30,35,37,38]$ for this definition. The following result is a converse theorem on (global) exponential stability, which guarantees the existence of a Lyapunov function estimated by quadratic form $\|\mathbf{x}\|^{2}$ (see $[30,31,34$, $35])$.

Theorem B Suppose that for any $\alpha>0$, there exists an $L(\alpha)>0$ such that

$$
\|\mathbf{f}(t, \mathbf{x})-\mathbf{f}(t, \mathbf{y})\| \leq L(\alpha)\|\mathbf{x}-\mathbf{y}\|
$$

for $(t, \mathbf{x}),(t, \mathbf{y}) \in I \times S_{\alpha}$, where $L(\alpha)$ is independent of $t \in I$. If the zero solution of (1.4) is globally exponentially stable, then there exist three positive constants $\beta_{1}(\alpha), \beta_{2}(\alpha), \beta_{3}$ and a Lyapunov function $V(t, \mathbf{x})$ defined on $I \times S_{\alpha}$ which satisfies the following conditions:

(i) $\beta_{1}(\alpha)\|\mathbf{x}\|^{2} \leq V(t, \mathbf{x}) \leq \beta_{2}(\alpha)\|\mathbf{x}\|^{2}$;

(ii) $\dot{V}_{(1.4)}(t, \mathbf{x}) \leq-\beta_{3}\|\mathbf{x}\|^{2}$,

where $\alpha$ is a number given in the definition of global exponential stability.

In addition, another type converse theorem on (global) exponential stability can also be found in the classical books $[28,29]$.

Theorem C Suppose that $\mathbf{f}(t, \mathbf{x})$ satisfy locally Lipschitz condition. If the zero solution of (1.4) is globally exponentially stable, then there exists a Lyapunov function $V(t, \mathbf{x})$ defined on $I \times S_{\alpha}$ which satisfies the following conditions: 
(i) $\|\mathbf{x}\| \leq V(t, \mathbf{x}) \leq \beta(\alpha)\|\mathbf{x}\|$;

(ii) $\dot{V}_{(1.4)}(t, \mathbf{x}) \leq-k \lambda V(t, \mathbf{x})$, where $0<k<1$;

(iii) there exists an $L(t, \alpha)>0$ such that $|V(t, \mathbf{x})-V(t, \mathbf{y})| \leq L(t, \alpha)\|\mathbf{x}-\mathbf{y}\|$ for $(t, \mathbf{x}),(t, \mathbf{y}) \in I \times S_{\alpha}$,

where $\alpha, \beta$, and $\lambda$ are numbers given in the definition of global exponential stability.

When restricted to the case of the linear system (1.3), the following facts are known (see ([28], pp.44-45), ([29], p.78) and ([37], p.155)).

Theorem D If the zero solution of (1.3) is exponentially stable, then it is globally exponentially stable. In this case, we can find $a \beta>0$ independent of $\alpha$ in the definition of globally exponentially stable.

From Theorems A and D, for the linear differential system (1.3), uniform asymptotic stability and global exponential stability are equivalent. In the case of the linear system (1.3), the following converse theorem on (global) exponential stability is known (see ([28], pp.92-93), ([29], p.105) and ([32], p.327)).

Theorem E Suppose that the zero solution of (1.3) is globally exponentially stable, i.e., there exist $a \lambda>0$ and $a \beta>0$ such that

$$
\left\|\mathbf{x}\left(t ; t_{0}, \mathbf{x}_{0}\right)\right\| \leq \beta e^{-\lambda\left(t-t_{0}\right)}\left\|\mathbf{x}_{0}\right\|
$$

for all $t \geq t_{0}$. Then there exists a Lyapunov function $V(t, \mathbf{x})$ defined on $I \times \mathbb{R}^{n}$ which satisfies the following conditions:

(i) $\|\mathbf{x}\| \leq V(t, \mathbf{x}) \leq \beta\|\mathbf{x}\|$;

(ii) $\dot{V}_{(1.4)}(t, \mathbf{x}) \leq-\lambda V(t, \mathbf{x})$;

(iii) $|V(t, \mathbf{x})-V(t, \mathbf{y})| \leq \beta\|\mathbf{x}-\mathbf{y}\|$ for $(t, \mathbf{x}),(t, \mathbf{y}) \in I \times \mathbb{R}^{n}$.

To present our result, we give some definitions of stability and its equivalent conditions in the next section. Also, we give a proposition which is the most important property for the proof of Theorem 1.1. In Section 3, we state the proof of Theorem 1.1. In Section 4, we give a natural generalization of Theorem $\mathrm{D}$ with $n=2$. In the final section, we present the converse theorems for half-linear system (1.1), for comparison with Theorems B, C, and E.

\section{Definitions and lemmas}

We now give some definitions about the zero solution $\mathbf{x}\left(t ; t_{0}, \mathbf{0}\right) \equiv \mathbf{0}$ of (1.1). The zero solution is said to be uniformly attractive if there exists a $\delta_{0}>0$ and, for every $\varepsilon>0$, there exists a $T(\varepsilon)>0$ such that $t_{0} \in I$ and $\left\|\mathbf{x}_{0}\right\|<\delta_{0}$ imply $\left\|\mathbf{x}\left(t ; t_{0}, \mathbf{x}_{0}\right)\right\|<\varepsilon$ for all $t \geq t_{0}+T(\varepsilon)$. The zero solution of (1.1) is said to be uniformly stable if, for any $\varepsilon>0$, there exists a $\delta(\varepsilon)>0$ such that $t_{0} \in I$ and $\left\|\mathbf{x}_{0}\right\|<\delta(\varepsilon)$ imply $\left\|\mathbf{x}\left(t ; t_{0}, \mathbf{x}_{0}\right)\right\|<\varepsilon$ for all $t \geq t_{0}$. The zero solution is uniformly asymptotically stable if it is uniformly attractive and is uniformly stable. The zero solution is said to be exponentially stable (or exponentially asymptotically stable); if there exists a $\lambda>0$ and, given any $\varepsilon>0$, there exists a $\delta(\varepsilon)>0$ such that $t_{0} \in I$ and $\left\|\mathbf{x}_{0}\right\|<\delta(\varepsilon)$ imply $\left\|\mathbf{x}\left(t ; t_{0}, \mathbf{x}_{0}\right)\right\| \leq \varepsilon e^{-\lambda\left(t-t_{0}\right)}$ for all $t \geq t_{0}$. For example, we can refer to the books and papers $[7,18,19,25-53]$ for those definitions. 
In this section, before giving the proof of Theorem 1.1, we prepare some lemmas. First, we will give the conditions which are equivalent to the above mentioned definitions. Some of these conditions are applicable to the proof of Theorem 1.1. For $\mathbf{x}=(x, y) \in \mathbb{R}^{2}$ and $p \geq 1$, we define a norm $\|\mathbf{x}\|_{p}$ by $\sqrt[p]{|x|^{p}+|y|^{p}}$. This norm is often called the 'Hölder norm' or the 'p-norm' (see [30, 31, 37, 54, 55]).

Lemma 2.1 The zero solution of (1.1) is uniformly attractive if and only if there exists a $\gamma_{0}>0$ and, for every $\rho>0$, there exists an $S(\rho)>0$ such that $t_{0} \in I$ and $\left\|\left(x_{0}, \phi_{p^{*}}\left(y_{0}\right)\right)\right\|_{p}<\gamma_{0}$ imply

$$
\left\|\left(x\left(t ; t_{0}, x_{0}, y_{0}\right), \phi_{p^{*}}\left(y\left(t ; t_{0}, x_{0}, y_{0}\right)\right)\right)\right\|_{p}<\rho
$$

for all $t \geq t_{0}+S(\rho)$.

Proof First we prove the necessity. We suppose that the zero solution of (1.1) is uniformly attractive. That is, there exists a $\delta_{0}>0$ and, for every $\varepsilon>0$, there exists a $T(\varepsilon)>0$ such that $t_{0} \in I$ and $\left\|\left(x_{0}, y_{0}\right)\right\|<\delta_{0}$ imply $\left\|\left(x\left(t ; t_{0}, x_{0}, y_{0}\right), y\left(t ; t_{0}, x_{0}, y_{0}\right)\right)\right\|<\varepsilon$ for all $t \geq t_{0}+T$. Let

$$
\bar{p}=\max \left\{p, p^{*}\right\} \quad \text { and } \quad \gamma_{0}=\min \left\{1,\left(\frac{\delta_{0}}{\sqrt{2}}\right)^{\frac{\bar{p}}{p}}\right\}
$$

For every $0<\rho<1$, we determine $\varepsilon=\rho^{p} / 2$ and $S(\rho)=T\left(\rho^{p} / 2\right)$. We consider the solution $\left(x\left(t ; t_{0}, x_{0}, y_{0}\right), y\left(t ; t_{0}, x_{0}, y_{0}\right)\right)$ of (1.1) with $t_{0} \in I$ and $\left\|\left(x_{0}, \phi_{p^{*}}\left(y_{0}\right)\right)\right\|_{p}<\gamma_{0}$. From $\sqrt[p]{\left|x_{0}\right|^{p}+\left|y_{0}\right|^{p^{*}}}=\left\|\left(x_{0}, \phi_{p^{*}}\left(y_{0}\right)\right)\right\|_{p}<\gamma_{0}$ it follows that

$$
\left|x_{0}\right|<\gamma_{0} \text { and }\left|y_{0}\right|<\gamma_{0} \frac{p}{p^{*}} \text {. }
$$

Hence, combining this estimation with $0<\gamma_{0} \leq 1 \leq \bar{p} / p$ and $\bar{p} / p^{*} \geq 1$, we obtain

$$
\begin{aligned}
\left\|\left(x_{0}, y_{0}\right)\right\| & =\sqrt{x_{0}^{2}+y_{0}^{2}}<\sqrt{\gamma_{0}^{2}+\gamma_{0}^{\frac{2 p}{p^{*}}}} \\
& =\sqrt{\min \left\{1,\left(\frac{\delta_{0}}{\sqrt{2}}\right)^{\frac{2 \bar{p}}{p}}\right\}+\min \left\{1,\left(\frac{\delta_{0}}{\sqrt{2}}\right)^{\frac{2 \bar{p}}{p^{*}}}\right\}} \\
& \leq \sqrt{2 \min \left\{1,\left(\frac{\delta_{0}}{\sqrt{2}}\right)^{2}\right\}} \leq \delta_{0},
\end{aligned}
$$

and, therefore,

$$
\left\|\left(x\left(t ; t_{0}, x_{0}, y_{0}\right), y\left(t ; t_{0}, x_{0}, y_{0}\right)\right)\right\|<\varepsilon=\frac{\rho^{p}}{2}
$$

for $t \geq t_{0}+S$. Taking into account that this inequality and $0<\rho^{p} / 2<1<p$ and $p^{*}>1$ hold, we have

$$
\left|x\left(t ; t_{0}, x_{0}, y_{0}\right)\right|^{p}<\left(\frac{\rho^{p}}{2}\right)^{p}<\frac{\rho^{p}}{2} \quad \text { and } \quad\left|y\left(t ; t_{0}, x_{0}, y_{0}\right)\right|^{p^{*}}<\left(\frac{\rho^{p}}{2}\right)^{p^{*}}<\frac{\rho^{p}}{2}
$$


for $t \geq t_{0}+S$. From these inequalities, we see that

$$
\left\|\left(x\left(t ; t_{0}, x_{0}, y_{0}\right), \phi_{p^{*}}\left(y\left(t ; t_{0}, x_{0}, y_{0}\right)\right)\right)\right\|_{p}<\sqrt[p]{\frac{\rho^{p}}{2}+\frac{\rho^{p}}{2}}=\rho
$$

for $t \geq t_{0}+S$.

Conversely, we prove the sufficiency. We suppose that there exists a $\gamma_{0}>0$ and, for every $\rho>0$, there exists an $S(\rho)>0$ such that $t_{0} \in I$ and $\left\|\left(x_{0}, \phi_{p^{*}}\left(y_{0}\right)\right)\right\|_{p}<\gamma_{0}$ imply $\left\|\left(x\left(t ; t_{0}, x_{0}, y_{0}\right), \phi_{p^{*}}\left(y\left(t ; t_{0}, x_{0}, y_{0}\right)\right)\right)\right\|_{p}<\rho$ for all $t \geq t_{0}+S$. Let

$$
\delta_{0}=\min \left\{1, \frac{\gamma_{0}^{p}}{2}\right\}
$$

For every $0<\varepsilon<1$, we determine $\rho=(\varepsilon / \sqrt{2})^{\bar{p} / p}$ and $T(\varepsilon)=S\left((\varepsilon / \sqrt{2})^{\bar{p} / p}\right)$. We consider the solution $\left(x\left(t ; t_{0}, x_{0}, y_{0}\right), y\left(t ; t_{0}, x_{0}, y_{0}\right)\right)$ of $(1.1)$ with $t_{0} \in I$ and $\left\|\left(x_{0}, y_{0}\right)\right\|<\delta_{0}$. From $\left\|\left(x_{0}, y_{0}\right)\right\|<\delta_{0}$ it follows that

$$
\left|x_{0}\right|<\delta_{0} \quad \text { and } \quad\left|y_{0}\right|<\delta_{0}
$$

Hence, combining this estimation with $0<\delta_{0} \leq 1<p$ and $p^{*}>1$, we obtain

$$
\begin{aligned}
\left\|\left(x_{0}, \phi_{p^{*}}\left(y_{0}\right)\right)\right\|_{p} & <\sqrt[p]{\delta_{0}^{p}+\delta_{0}^{p^{*}}}=\sqrt[p]{\min \left\{1,\left(\frac{\gamma_{0}{ }^{p}}{2}\right)^{p}\right\}+\min \left\{1,\left(\frac{\gamma_{0}{ }^{p}}{2}\right)^{p^{*}}\right\}} \\
& \leq \sqrt[p]{2 \min \left\{1, \frac{\gamma_{0} p}{2}\right\}} \leq \gamma_{0},
\end{aligned}
$$

and, therefore,

$$
\left\|\left(x\left(t ; t_{0}, x_{0}, y_{0}\right), \phi_{p^{*}}\left(y\left(t ; t_{0}, x_{0}, y_{0}\right)\right)\right)\right\|_{p}<\rho=\left(\frac{\varepsilon}{\sqrt{2}}\right)^{\frac{\bar{p}}{p}}
$$

for $t \geq t_{0}+T$. Taking into account that this inequality and $0<\varepsilon^{2} / 2<1 \leq \bar{p} / p$, and $\bar{p} / p^{*} \geq 1$ hold, we have

$$
x^{2}\left(t ; t_{0}, x_{0}, y_{0}\right)<\left(\frac{\varepsilon^{2}}{2}\right)^{\frac{\bar{p}}{p}} \leq \frac{\varepsilon^{2}}{2} \quad \text { and } \quad y^{2}\left(t ; t_{0}, x_{0}, y_{0}\right)<\left(\frac{\varepsilon^{2}}{2}\right)^{\frac{\bar{p}}{p^{*}}} \leq \frac{\varepsilon^{2}}{2}
$$

for $t \geq t_{0}+T$. From these inequalities, we see that

$$
\left\|\left(x\left(t ; t_{0}, x_{0}, y_{0}\right), y\left(t ; t_{0}, x_{0}, y_{0}\right)\right)\right\|<\varepsilon
$$

for $t \geq t_{0}+T$. This completes the proof of Lemma 2.1.

By using the same arguments as in Lemma 2.1, we can prove the following lemma.

Lemma 2.2 The zero solution of (1.1) is uniformly stable if and only if, for any $\rho>0$, there exists a $\gamma(\rho)>0$ such that $t_{0} \in I$ and $\left\|\left(x_{0}, \phi_{p^{*}}\left(y_{0}\right)\right)\right\|_{p}<\gamma(\rho)$ imply

$$
\left\|\left(x\left(t ; t_{0}, x_{0}, y_{0}\right), \phi_{p^{*}}\left(y\left(t ; t_{0}, x_{0}, y_{0}\right)\right)\right)\right\|_{p}<\rho
$$

for all $t \geq t_{0}$. 
Proof First we prove the necessity. We suppose that the zero solution of (1.1) is uniformly stable. That is, for any $\varepsilon>0$, there exists a $\delta(\varepsilon)>0$ such that $t_{0} \in I$ and $\left\|\left(x_{0}, y_{0}\right)\right\|<\delta(\varepsilon)$ imply $\left\|\left(x\left(t ; t_{0}, x_{0}, y_{0}\right), y\left(t ; t_{0}, x_{0}, y_{0}\right)\right)\right\|<\varepsilon$ for all $t \geq t_{0}$. For every $0<\rho<1$, we determine an $\varepsilon=\rho^{p} / 2$. Let

$$
\bar{p}=\max \left\{p, p^{*}\right\} \quad \text { and } \quad \gamma(\rho)=\min \left\{1,\left(\frac{1}{\sqrt{2}} \delta\left(\frac{\rho^{p}}{2}\right)\right)^{\frac{\bar{p}}{p}}\right\}
$$

We consider the solution $\left(x\left(t ; t_{0}, x_{0}, y_{0}\right), y\left(t ; t_{0}, x_{0}, y_{0}\right)\right)$ of (1.1) with $t_{0} \in I$ and $\|\left(x_{0}\right.$, $\left.\phi_{p^{*}}\left(y_{0}\right)\right) \|_{p}<\gamma$. From $\sqrt[p]{\left|x_{0}\right|^{p}+\left|y_{0}\right|^{p^{*}}}=\left\|\left(x_{0}, \phi_{p^{*}}\left(y_{0}\right)\right)\right\|_{p}<\gamma$ it follows that

$$
\left|x_{0}\right|<\gamma \quad \text { and } \quad\left|y_{0}\right|<\gamma^{\frac{p}{p^{*}}}
$$

Hence, combining this estimation with $0<\gamma \leq 1 \leq \bar{p} / p$ and $\bar{p} / p^{*} \geq 1$, we obtain

$$
\begin{aligned}
\left\|\left(x_{0}, y_{0}\right)\right\| & =\sqrt{x_{0}^{2}+y_{0}^{2}}<\sqrt{\gamma^{2}+\gamma^{\frac{2 p}{p^{*}}}} \\
& =\sqrt{\min \left\{1,\left(\frac{\delta}{\sqrt{2}}\right)^{\frac{2 \bar{p}}{p}}\right\}+\min \left\{1,\left(\frac{\delta}{\sqrt{2}}\right)^{\frac{2 \bar{p}}{p^{*}}}\right\}} \\
& \leq \sqrt{2 \min \left\{1,\left(\frac{\delta}{\sqrt{2}}\right)^{2}\right\}} \leq \delta,
\end{aligned}
$$

and, therefore,

$$
\left\|\left(x\left(t ; t_{0}, x_{0}, y_{0}\right), y\left(t ; t_{0}, x_{0}, y_{0}\right)\right)\right\|<\varepsilon=\frac{\rho^{p}}{2}
$$

for $t \geq t_{0}$. Taking into account that this inequality and $0<\rho^{p} / 2<1<p$ and $p^{*}>1$ hold, we have

$$
\left|x\left(t ; t_{0}, x_{0}, y_{0}\right)\right|^{p}<\left(\frac{\rho^{p}}{2}\right)^{p}<\frac{\rho^{p}}{2} \quad \text { and } \quad\left|y\left(t ; t_{0}, x_{0}, y_{0}\right)\right|^{p^{*}}<\left(\frac{\rho^{p}}{2}\right)^{p^{*}}<\frac{\rho^{p}}{2}
$$

for $t \geq t_{0}$. From these inequalities, we see that

$$
\left\|\left(x\left(t ; t_{0}, x_{0}, y_{0}\right), \phi_{p^{*}}\left(y\left(t ; t_{0}, x_{0}, y_{0}\right)\right)\right)\right\|_{p}<\sqrt[p]{\frac{\rho^{p}}{2}+\frac{\rho^{p}}{2}}=\rho
$$

for $t \geq t_{0}$.

Conversely, we prove the sufficiency. We suppose that for any $\rho>0$, there exists a $\gamma(\rho)>$ 0 such that $t_{0} \in I$ and $\left\|\left(x_{0}, \phi_{p^{*}}\left(y_{0}\right)\right)\right\|_{p}<\gamma(\rho)$ imply

$$
\left\|\left(x\left(t ; t_{0}, x_{0}, y_{0}\right), \phi_{p^{*}}\left(y\left(t ; t_{0}, x_{0}, y_{0}\right)\right)\right)\right\|_{p}<\rho
$$

for all $t \geq t_{0}$. For every $0<\varepsilon<1$, we determine a $\rho=(\varepsilon / \sqrt{2})^{\bar{p} / p}$. Let

$$
\delta(\varepsilon)=\min \left\{1, \frac{1}{2} \gamma^{p}\left(\left(\frac{\varepsilon}{\sqrt{2}}\right)^{\frac{\bar{p}}{p}}\right)\right\} .
$$


We consider the solution $\left(x\left(t ; t_{0}, x_{0}, y_{0}\right), y\left(t ; t_{0}, x_{0}, y_{0}\right)\right)$ of $(1.1)$ with $t_{0} \in I$ and $\left\|\left(x_{0}, y_{0}\right)\right\|<\delta$. From $\left\|\left(x_{0}, y_{0}\right)\right\|<\delta$ it follows that

$$
\left|x_{0}\right|<\delta \text { and }\left|y_{0}\right|<\delta \text {. }
$$

Hence, combining this estimation with $0<\delta \leq 1<p$ and $p^{*}>1$, we obtain

$$
\begin{aligned}
\left\|\left(x_{0}, \phi_{p^{*}}\left(y_{0}\right)\right)\right\|_{p} & <\sqrt[p]{\delta^{p}+\delta^{p^{*}}}=\sqrt[p]{\min \left\{1,\left(\frac{\gamma^{p}}{2}\right)^{p}\right\}+\min \left\{1,\left(\frac{\gamma^{p}}{2}\right)^{p^{*}}\right\}} \\
& \leq \sqrt[p]{2 \min \left\{1, \frac{\gamma^{p}}{2}\right\}} \leq \gamma,
\end{aligned}
$$

and, therefore,

$$
\left\|\left(x\left(t ; t_{0}, x_{0}, y_{0}\right), \phi_{p^{*}}\left(y\left(t ; t_{0}, x_{0}, y_{0}\right)\right)\right)\right\|_{p}<\rho=\left(\frac{\varepsilon}{\sqrt{2}}\right)^{\frac{\bar{p}}{p}}
$$

for $t \geq t_{0}$. Taking into account that this inequality and $0<\varepsilon^{2} / 2<1 \leq \bar{p} / p$ and $\bar{p} / p^{*} \geq 1$ hold, we have

$$
x^{2}\left(t ; t_{0}, x_{0}, y_{0}\right)<\left(\frac{\varepsilon^{2}}{2}\right)^{\frac{\bar{p}}{p}} \leq \frac{\varepsilon^{2}}{2} \quad \text { and } \quad y^{2}\left(t ; t_{0}, x_{0}, y_{0}\right)<\left(\frac{\varepsilon^{2}}{2}\right)^{\frac{\bar{p}}{p^{*}}} \leq \frac{\varepsilon^{2}}{2}
$$

for $t \geq t_{0}$. From these inequalities, we see that

$$
\left\|\left(x\left(t ; t_{0}, x_{0}, y_{0}\right), y\left(t ; t_{0}, x_{0}, y_{0}\right)\right)\right\|<\varepsilon
$$

for $t \geq t_{0}$. This completes the proof of Lemma 2.2.

Furthermore, by using the same arguments as in Lemmas 2.1 and 2.2, we have the following result.

Lemma 2.3 The zero solution of (1.1) is exponentially stable if and only if there exists a $\mu>0$ and, given any $\rho>0$, there exists a $\gamma(\rho)>0$ such that $t_{0} \in I$ and $\left\|\left(x_{0}, \phi_{p^{*}}\left(y_{0}\right)\right)\right\|_{p}<$ $\gamma(\rho)$ imply

$$
\left\|\left(x\left(t ; t_{0}, x_{0}, y_{0}\right), \phi_{p^{*}}\left(y\left(t ; t_{0}, x_{0}, y_{0}\right)\right)\right)\right\|_{p} \leq \rho e^{-\mu\left(t-t_{0}\right)}
$$

for all $t \geq t_{0}$.

Proof First we prove the necessity. We suppose that the zero solution of (1.1) is exponentially stable. That is, there exists a $\lambda>0$ and, given any $\varepsilon>0$, there exists a $\delta(\varepsilon)>0$ such that $t_{0} \in I$ and $\left\|\left(x_{0}, y_{0}\right)\right\|<\delta(\varepsilon)$ imply $\left\|\left(x\left(t ; t_{0}, x_{0}, y_{0}\right), y\left(t ; t_{0}, x_{0}, y_{0}\right)\right)\right\| \leq \varepsilon e^{-\lambda\left(t-t_{0}\right)}$ for all $t \geq t_{0}$. Let $\mu=\lambda / p$. For every $0<\rho<1$, we determine an $\varepsilon=\rho^{p} / 2$. Let

$$
\bar{p}=\max \left\{p, p^{*}\right\} \quad \text { and } \quad \gamma(\rho)=\min \left\{1,\left(\frac{1}{\sqrt{2}} \delta\left(\frac{\rho^{p}}{2}\right)\right)^{\frac{\bar{p}}{p}}\right\} .
$$


We consider the solution $\left(x\left(t ; t_{0}, x_{0}, y_{0}\right), y\left(t ; t_{0}, x_{0}, y_{0}\right)\right)$ of (1.1) with $t_{0} \in I$ and $\|\left(x_{0}\right.$, $\left.\phi_{p^{*}}\left(y_{0}\right)\right) \|_{p}<\gamma$. From $\sqrt[p]{\left|x_{0}\right|^{p}+\left|y_{0}\right|^{p^{*}}}=\left\|\left(x_{0}, \phi_{p^{*}}\left(y_{0}\right)\right)\right\|_{p}<\gamma$ it follows that

$$
\left|x_{0}\right|<\gamma \quad \text { and } \quad\left|y_{0}\right|<\gamma^{\frac{p}{p^{*}}}
$$

Hence, combining this estimation with $0<\gamma \leq 1 \leq \bar{p} / p$ and $\bar{p} / p^{*} \geq 1$ we obtain

$$
\begin{aligned}
\left\|\left(x_{0}, y_{0}\right)\right\| & =\sqrt{x_{0}^{2}+y_{0}^{2}}<\sqrt{\gamma^{2}+\gamma^{\frac{2 p}{p^{*}}}} \\
& =\sqrt{\min \left\{1,\left(\frac{\delta}{\sqrt{2}}\right)^{\frac{2 \bar{p}}{p}}\right\}+\min \left\{1,\left(\frac{\delta}{\sqrt{2}}\right)^{\frac{2 \bar{p}}{p^{*}}}\right\}} \\
& \leq \sqrt{2 \min \left\{1,\left(\frac{\delta}{\sqrt{2}}\right)^{2}\right\}} \leq \delta,
\end{aligned}
$$

and, therefore,

$$
\left\|\left(x\left(t ; t_{0}, x_{0}, y_{0}\right), y\left(t ; t_{0}, x_{0}, y_{0}\right)\right)\right\| \leq \varepsilon e^{-\lambda\left(t-t_{0}\right)}=\frac{\rho^{p}}{2} e^{-p \mu\left(t-t_{0}\right)}
$$

for $t \geq t_{0}$. Taking into account that this inequality and

$$
0<\frac{\rho^{p}}{2} e^{-p \mu\left(t-t_{0}\right)} \leq \frac{\rho^{p}}{2}<1<p \quad \text { and } \quad 1<p^{*}
$$

hold, we have

$$
\left|x\left(t ; t_{0}, x_{0}, y_{0}\right)\right|^{p} \leq\left(\frac{\rho^{p}}{2} e^{-p \mu\left(t-t_{0}\right)}\right)^{p}<\frac{\rho^{p}}{2} e^{-p \mu\left(t-t_{0}\right)}
$$

and

$$
\left|y\left(t ; t_{0}, x_{0}, y_{0}\right)\right|^{p^{*}} \leq\left(\frac{\rho^{p}}{2} e^{-p \mu\left(t-t_{0}\right)}\right)^{p^{*}}<\frac{\rho^{p}}{2} e^{-p \mu\left(t-t_{0}\right)}
$$

for $t \geq t_{0}$. From these inequalities, we see that

$$
\left\|\left(x\left(t ; t_{0}, x_{0}, y_{0}\right), \phi_{p^{*}}\left(y\left(t ; t_{0}, x_{0}, y_{0}\right)\right)\right)\right\|_{p}<\rho e^{-\mu\left(t-t_{0}\right)}
$$

for $t \geq t_{0}$.

Conversely, we prove the sufficiency. We suppose that there exists a $\mu>0$ and, given any $\rho>0$, there exists a $\gamma(\rho)>0$ such that $t_{0} \in I$ and $\left\|\left(x_{0}, \phi_{p^{*}}\left(y_{0}\right)\right)\right\|_{p}<\gamma(\rho)$ imply

$$
\left\|\left(x\left(t ; t_{0}, x_{0}, y_{0}\right), \phi_{p^{*}}\left(y\left(t ; t_{0}, x_{0}, y_{0}\right)\right)\right)\right\|_{p} \leq \rho e^{-\mu\left(t-t_{0}\right)}
$$

for all $t \geq t_{0}$. Let $\lambda=\mu p / \bar{p}$. For every $0<\varepsilon<1$, we determine a $\rho=(\varepsilon / \sqrt{2})^{\bar{p} / p}$. Let

$$
\delta(\varepsilon)=\min \left\{1, \frac{1}{2} \gamma^{p}\left(\left(\frac{\varepsilon}{\sqrt{2}}\right)^{\frac{\bar{p}}{p}}\right)\right\} .
$$


We consider the solution $\left(x\left(t ; t_{0}, x_{0}, y_{0}\right), y\left(t ; t_{0}, x_{0}, y_{0}\right)\right)$ of $(1.1)$ with $t_{0} \in I$ and $\left\|\left(x_{0}, y_{0}\right)\right\|<\delta$. From $\left\|\left(x_{0}, y_{0}\right)\right\|<\delta$ it follows that

$$
\left|x_{0}\right|<\delta \text { and }\left|y_{0}\right|<\delta
$$

Hence, combining this estimation with $0<\delta \leq 1<p$ and $p^{*}>1$, we obtain

$$
\begin{aligned}
\left\|\left(x_{0}, \phi_{p^{*}}\left(y_{0}\right)\right)\right\|_{p} & <\sqrt[p]{\delta^{p}+\delta^{p^{*}}}=\sqrt[p]{\min \left\{1,\left(\frac{\gamma^{p}}{2}\right)^{p}\right\}+\min \left\{1,\left(\frac{\gamma^{p}}{2}\right)^{p^{*}}\right\}} \\
& \leq \sqrt[p]{2 \min \left\{1, \frac{\gamma^{p}}{2}\right\}} \leq \gamma
\end{aligned}
$$

and, therefore,

$$
\left\|\left(x\left(t ; t_{0}, x_{0}, y_{0}\right), \phi_{p^{*}}\left(y\left(t ; t_{0}, x_{0}, y_{0}\right)\right)\right)\right\|_{p} \leq \rho e^{-\mu\left(t-t_{0}\right)}=\left(\frac{\varepsilon}{\sqrt{2}}\right)^{\frac{\bar{p}}{p}} e^{-\frac{\bar{p} \lambda}{p}\left(t-t_{0}\right)}
$$

for $t \geq t_{0}$. Taking into account that this inequality and

$$
0<\frac{\varepsilon}{\sqrt{2}} e^{-\lambda\left(t-t_{0}\right)} \leq \frac{\varepsilon}{\sqrt{2}}<1 \leq \frac{\bar{p}}{p} \quad \text { and } \quad 1 \leq \frac{\bar{p}}{p^{*}}
$$

hold, we have

$$
\left|x\left(t ; t_{0}, x_{0}, y_{0}\right)\right| \leq\left(\frac{\varepsilon}{\sqrt{2}} e^{-\lambda\left(t-t_{0}\right)}\right)^{\frac{\bar{p}}{p}} \leq \frac{\varepsilon}{\sqrt{2}} e^{-\lambda\left(t-t_{0}\right)}
$$

and

$$
\left|y\left(t ; t_{0}, x_{0}, y_{0}\right)\right| \leq\left(\frac{\varepsilon}{\sqrt{2}} e^{-\lambda\left(t-t_{0}\right)}\right)^{\frac{\bar{p}}{p^{*}}} \leq \frac{\varepsilon}{\sqrt{2}} e^{-\lambda\left(t-t_{0}\right)}
$$

for $t \geq t_{0}$. From these inequalities, we see that

$$
\left\|\left(x\left(t ; t_{0}, x_{0}, y_{0}\right), y\left(t ; t_{0}, x_{0}, y_{0}\right)\right)\right\| \leq \varepsilon e^{-\lambda\left(t-t_{0}\right)}
$$

for $t \geq t_{0}$. This completes the proof of Lemma 2.3.

In the special case in which $p=2$, (1.1) becomes the linear system. As is well known, the solution space of the linear system is homogeneous and additive. On the other hand, in the general case in which $p \neq 2$, the solution space of (1.1) is not homogeneous or additive. However, we can show that (1.1) has a homogeneous-like property on the solution space.

Lemma 2.4 If $(x(t), y(t))$ is a solution of (1.1) passing through a point $\left(x_{0}, y_{0}\right) \in \mathbb{R}^{2}$ at $t=$ $t_{0} \in I$, then $\left(c x(t), \phi_{p}(c) y(t)\right)$ is also a solution of (1.1) passing through a point $\left(c x_{0}, \phi_{p}(c) y_{0}\right) \in$ $\mathbb{R}^{2}$ at $t=t_{0}$ for any $c \in \mathbb{R}$. 
Proof We consider the solution $(x(t), y(t))$ of (1.1) passing through a point $\left(x_{0}, y_{0}\right)$ at $t=t_{0}$. Let $\tilde{x}(t)=c x(t)$ and $\tilde{y}(t)=\phi_{p}(c) y(t)$ with $c \in \mathbb{R}$. It is clear that $\left(\tilde{x}\left(t_{0}\right), \tilde{y}\left(t_{0}\right)\right)=\left(c x_{0}, \phi_{p}(c) y_{0}\right)$ is satisfied. Since $\phi_{p^{*}}$ is the inverse function of $\phi_{p}$, we have

$$
\tilde{x}^{\prime}(t)=a_{11}(t) c x(t)+a_{12}(t) \phi_{p^{*}}\left(\phi_{p}(c) y(t)\right)=a_{11}(t) \tilde{x}(t)+a_{12}(t) \phi_{p^{*}}(\tilde{y}(t))
$$

and

$$
\tilde{y}^{\prime}(t)=a_{21}(t) \phi_{p}(c x(t))+a_{22}(t) \phi_{p}(c) y(t)=a_{21}(t) \phi_{p}(\tilde{x}(t))+a_{22}(t) \tilde{y}(t) .
$$

We therefore conclude that $\left(c x(t), \phi_{p}(c) y(t)\right)$ is also a solution of (1.1) passing through a point $\left(c x_{0}, \phi_{p}(c) y_{0}\right)$ at $t=t_{0}$.

We state the following proposition, which is the most important property for the proof of Theorem 1.1.

Proposition 2.5 If the zero solution of (1.1) is uniformly attractive, then there exists a $\gamma_{0}>$ 0 and, for every $v>1$, there exists a $T(v)>0$ such that $t_{0} \in I$ and $\left\|\left(x_{0}, \phi_{p^{*}}\left(y_{0}\right)\right)\right\|_{p}<\gamma_{0} v^{-(k-1)}$ imply

$$
\left\|\left(x\left(t ; t_{0}+(k-1) T(v), x_{0}, y_{0}\right), \phi_{p^{*}}\left(y\left(t ; t_{0}+(k-1) T(v), x_{0}, y_{0}\right)\right)\right)\right\|_{p}<\gamma_{0} v^{-k}
$$

for all $t \geq t_{0}+k T(v)$ and $k \in \mathbb{N}$.

Proof By using the assumption and Lemma 2.1, there exists a $\gamma_{0}>0$ and, for every $v>1$, there exists an $S\left(\gamma_{0} / \nu\right)>0$ such that $\tau \geq 0$ and $\left\|\left(\xi, \phi_{p^{*}}(\eta)\right)\right\|_{p}<\gamma_{0}$ imply

$$
\left\|\left(x(t ; \tau, \xi, \eta), \phi_{p^{*}}(y(t ; \tau, \xi, \eta))\right)\right\|_{p}<\frac{\gamma_{0}}{v}
$$

for all $t \geq \tau+S$.

Let $T(v)=S\left(\gamma_{0} / v\right)$. We consider the solution

$$
\left(x\left(t ; t_{0}+(k-1) T, x_{0}, y_{0}\right), y\left(t ; t_{0}+(k-1) T, x_{0}, y_{0}\right)\right)
$$

of (1.1) with $t_{0} \in I$ and $\left\|\left(x_{0}, \phi_{p^{*}}\left(y_{0}\right)\right)\right\|_{p}<\gamma_{0} v^{-(k-1)}$. From Lemma 2.4, we conclude that

$$
\left(v^{k-1} x\left(t ; t_{0}+(k-1) T, x_{0}, y_{0}\right), \phi_{p}\left(v^{k-1}\right) y\left(t ; t_{0}+(k-1) T, x_{0}, y_{0}\right)\right)
$$

is also a solution of (1.1) passing through a point $\left(v^{k-1} x_{0}, \phi_{p}\left(v^{k-1}\right) y_{0}\right)$ at $t=t_{0}+(k-1) T$. Since

$$
\left\|\left(v^{k-1} x_{0}, v^{k-1} \phi_{p^{*}}\left(y_{0}\right)\right)\right\|_{p}=v^{k-1}\left\|\left(x_{0}, \phi_{p^{*}}\left(y_{0}\right)\right)\right\|_{p}<\gamma_{0}
$$

holds, we have

$$
\begin{aligned}
\frac{\gamma_{0}}{v} & >\left\|\left(v^{k-1} x\left(t ; t_{0}+(k-1) T, x_{0}, y_{0}\right), v^{k-1} \phi_{p^{*}}\left(y\left(t ; t_{0}+(k-1) T, x_{0}, y_{0}\right)\right)\right)\right\|_{p} \\
& =v^{k-1}\left\|\left(x\left(t ; t_{0}+(k-1) T, x_{0}, y_{0}\right), \phi_{p^{*}}\left(y\left(t ; t_{0}+(k-1) T, x_{0}, y_{0}\right)\right)\right)\right\|_{p}
\end{aligned}
$$


for all $t \geq t_{0}+(k-1) T+T=t_{0}+k T$. That is, we obtain

$$
\left\|\left(x\left(t ; t_{0}+(k-1) T, x_{0}, y_{0}\right), \phi_{p^{*}}\left(y\left(t ; t_{0}+(k-1) T, x_{0}, y_{0}\right)\right)\right)\right\|_{p}<\gamma_{0} v^{-k}
$$

for all $t \geq t_{0}+k T$. This completes the proof of Proposition 2.5.

\section{Exponential asymptotic stability}

In this section, we give the proof of the main theorem.

Proof of Theorem 1.1 By using uniform attractivity of (1.1) and Proposition 2.5, there exist a $\gamma_{0}>0$ and a $T(e)>0$ such that $t_{0} \in I$ and $\left\|\left(\xi, \phi_{p^{*}}(\eta)\right)\right\|_{p}<\gamma_{0} e^{-(k-1)}$ imply

$$
\left\|\left(x\left(t ; t_{0}+(k-1) T, \xi, \eta\right), \phi_{p^{*}}\left(y\left(t ; t_{0}+(k-1) T, \xi, \eta\right)\right)\right)\right\|_{p}<\gamma_{0} e^{-k}
$$

for all $t \geq t_{0}+k T$ and $k \in \mathbb{N}$.

Because of the uniform stability of (1.1) and Lemma 2.2, there exists a $\gamma\left(\gamma_{0}\right)>0$ such that $t_{0} \in I$ and $\left\|\left(\xi, \phi_{p^{*}}(\eta)\right)\right\|_{p}<\gamma$ imply

$$
\left\|\left(x\left(t ; t_{0}, \xi, \eta\right), \phi_{p^{*}}\left(y\left(t ; t_{0}, \xi, \eta\right)\right)\right)\right\|_{p}<\gamma_{0}
$$

for all $t \geq t_{0}$. Let $\lambda=1 / T$. For every $\varepsilon>0$, we determine a

$$
\delta(\varepsilon)=\frac{\gamma \varepsilon}{\gamma_{0} e}>0
$$

We now consider the solution $\left(x\left(t ; t_{0}, x_{0}, y_{0}\right), y\left(t ; t_{0}, x_{0}, y_{0}\right)\right)$ of $(1.1)$ with $t_{0} \in I$ and $\|\left(x_{0}\right.$, $\left.\phi_{p^{*}}\left(y_{0}\right)\right) \|_{p}<\delta$. For the sake of simplicity, let

$$
(x(t), y(t))=\left(x\left(t ; t_{0}, x_{0}, y_{0}\right), y\left(t ; t_{0}, x_{0}, y_{0}\right)\right) .
$$

Using Lemma 2.4, we can find a solution

$$
\left(\frac{\gamma_{0} e}{\varepsilon} x(t), \phi_{p}\left(\frac{\gamma_{0} e}{\varepsilon}\right) y(t)\right)
$$

of (1.1) passing through a point $\left(\left(\gamma_{0} e / \varepsilon\right) x_{0}, \phi_{p}\left(\gamma_{0} e / \varepsilon\right) y_{0}\right)$ at $t=t_{0}$. From $\delta=\gamma \varepsilon /\left(\gamma_{0} e\right)$, we see that

$$
\begin{aligned}
\left\|\left(\frac{\gamma_{0} e}{\varepsilon} x_{0}, \phi_{p^{*}}\left(\phi_{p}\left(\frac{\gamma_{0} e}{\varepsilon}\right) y_{0}\right)\right)\right\|_{p} & =\left\|\left(\frac{\gamma_{0} e}{\varepsilon} x_{0}, \frac{\gamma_{0} e}{\varepsilon} \phi_{p^{*}}\left(y_{0}\right)\right)\right\|_{p} \\
& =\frac{\gamma_{0} e}{\varepsilon}\left\|\left(x_{0}, \phi_{p^{*}}\left(y_{0}\right)\right)\right\|_{p}<\gamma
\end{aligned}
$$

at $t=t_{0}$. From this inequality and (3.2) with

$$
(\xi, \eta)=\left(\frac{\gamma_{0} e}{\varepsilon} x_{0}, \phi_{p}\left(\frac{\gamma_{0} e}{\varepsilon}\right) y_{0}\right),
$$


we have

$$
\begin{aligned}
\frac{\gamma_{0} e}{\varepsilon}\left\|\left(x(t), \phi_{p^{*}}(y(t))\right)\right\|_{p} & =\left\|\left(\frac{\gamma_{0} e}{\varepsilon} x(t), \frac{\gamma_{0} e}{\varepsilon} \phi_{p^{*}}(y(t))\right)\right\|_{p} \\
& =\left\|\left(\frac{\gamma_{0} e}{\varepsilon} x(t), \phi_{p^{*}}\left(\phi_{p}\left(\frac{\gamma_{0} e}{\varepsilon}\right) y(t)\right)\right)\right\|_{p}<\gamma_{0}
\end{aligned}
$$

for $t \geq t_{0}$. We therefore conclude that

$$
\left\|\left(x(t), \phi_{p^{*}}(y(t))\right)\right\|_{p}<\frac{\varepsilon}{e}
$$

for $t_{0} \leq t \leq t_{0}+T$. By using (3.3), we obtain

$$
\left\|\left(\frac{\gamma_{0} e}{\varepsilon} x_{0}, \phi_{p^{*}}\left(\phi_{p}\left(\frac{\gamma_{0} e}{\varepsilon}\right) y_{0}\right)\right)\right\|_{p}<\gamma_{0}
$$

at $t=t_{0}$. From this inequality and (3.1) with

$$
(\xi, \eta)=\left(\frac{\gamma_{0} e}{\varepsilon} x_{0}, \phi_{p}\left(\frac{\gamma_{0} e}{\varepsilon}\right) y_{0}\right), \quad k=1,
$$

we get

$$
\frac{\gamma_{0} e}{\varepsilon}\left\|\left(x(t), \phi_{p^{*}}(y(t))\right)\right\|_{p}=\left\|\left(\frac{\gamma_{0} e}{\varepsilon} x(t), \phi_{p^{*}}\left(\phi_{p}\left(\frac{\gamma_{0} e}{\varepsilon}\right) y(t)\right)\right)\right\|_{p}<\frac{\gamma_{0}}{e}
$$

for $t \geq t_{0}+T$. We therefore conclude that

$$
\left\|\left(x(t), \phi_{p^{*}}(y(t))\right)\right\|_{p}<\frac{\varepsilon}{e^{2}}
$$

for $t_{0}+T \leq t \leq t_{0}+2 T$. By using (3.4), we obtain

$$
\left\|\left(\frac{\gamma_{0} e}{\varepsilon} x\left(t_{0}+T\right), \phi_{p^{*}}\left(\phi_{p}\left(\frac{\gamma_{0} e}{\varepsilon}\right) y\left(t_{0}+T\right)\right)\right)\right\|_{p}<\frac{\gamma_{0}}{e}
$$

at $t=t_{0}+T$. From this inequality and (3.1) with

$$
(\xi, \eta)=\left(\frac{\gamma_{0} e}{\varepsilon} x\left(t_{0}+T\right), \phi_{p}\left(\frac{\gamma_{0} e}{\varepsilon}\right) y\left(t_{0}+T\right)\right), \quad k=2,
$$

we get

$$
\frac{\gamma_{0} e}{\varepsilon}\left\|\left(x(t), \phi_{p^{*}}(y(t))\right)\right\|_{p}=\left\|\left(\frac{\gamma_{0} e}{\varepsilon} x(t), \phi_{p^{*}}\left(\phi_{p}\left(\frac{\gamma_{0} e}{\varepsilon}\right) y(t)\right)\right)\right\|_{p}<\frac{\gamma_{0}}{e^{2}}
$$

for $t \geq t_{0}+2 T$. We therefore conclude that

$$
\left\|\left(x(t), \phi_{p^{*}}(y(t))\right)\right\|_{p}<\frac{\varepsilon}{e^{3}}
$$


for $t_{0}+2 T \leq t \leq t_{0}+3 T$. By means of the same process as in the above mentioned estimates, we see that

$$
\left\|\left(x(t), \phi_{p^{*}}(y(t))\right)\right\|_{p}<\varepsilon e^{-\kappa}
$$

for $t_{0}+(\kappa-1) T \leq t \leq t_{0}+\kappa T$ and $\kappa \in \mathbb{N}$. Hence, by $t \leq t_{0}+\kappa T$ we have

$$
-\kappa \leq-\frac{1}{T}\left(t-t_{0}\right)=-\lambda\left(t-t_{0}\right)
$$

and therefore

$$
\left\|\left(x(t), \phi_{p^{*}}(y(t))\right)\right\|_{p}<\varepsilon e^{-\kappa} \leq \varepsilon e^{-\lambda\left(t-t_{0}\right)}
$$

for $t_{0}+(\kappa-1) T \leq t \leq t_{0}+\kappa T$ and $\kappa \in \mathbb{N}$. Note that we can divide the interval $\left[t_{0}, t_{0}+\kappa T\right]$ as

$$
\left[t_{0}, t_{0}+\kappa T\right]=\bigcup_{n=1}^{\kappa}\left[t_{0}+(n-1) T, t_{0}+n T\right]
$$

for $\kappa \in \mathbb{N}$. Thus, it turns out that

$$
\left\|\left(x(t), \phi_{p^{*}}(y(t))\right)\right\|_{p} \leq \varepsilon e^{-\lambda\left(t-t_{0}\right)}
$$

for $t \geq t_{0}$. Using Lemma 2.3, we conclude that the zero solution of (1.1) is exponentially stable. This completes the proof of Theorem 1.1.

Note here that the proof of Theorem 1.1 does not require the uniqueness of solutions for the initial value problem.

\section{Global exponential stability}

Clearly concepts of above mentioned stability are local theory about the zero solution. In this section, we will discuss any initial disturbance and initial state. We consider the nonlinear scalar equation $x^{\prime}=-x+x^{2}$ (see [27], p.508). It is easy to see that the solution of this equation is given by

$$
x\left(t ; t_{0}, x_{0}\right)=\frac{x_{0}}{x_{0}-\left(x_{0}-1\right) e^{t-t_{0}}},
$$

where $t_{0} \in I$ and $x_{0} \in \mathbb{R}$. Clearly, $x\left(t ; t_{0}, 0\right) \equiv 0$ and $x\left(t ; t_{0}, 1\right) \equiv 1$ are the trivial solution. If $x_{0}<1$, then $x\left(t ; t_{0}, x_{0}\right) \rightarrow 0$ as $t \rightarrow \infty$. Moreover, for every $0<\varepsilon<1$, we choose $\delta(\varepsilon)=\varepsilon / 2$. If $\left|x_{0}\right|<\delta(\varepsilon)$, then we have

$$
\left|x\left(t ; t_{0}, x_{0}\right)\right| \leq \frac{\left|x_{0}\right| e^{-\left(t-t_{0}\right)}}{1-\left|x_{0}\right|\left(1-e^{-\left(t-t_{0}\right)}\right)}<\frac{\left|x_{0}\right| e^{-\left(t-t_{0}\right)}}{1-\left|x_{0}\right|}<\varepsilon e^{-\left(t-t_{0}\right)}
$$

for $t \geq t_{0}$. This means that the zero solution is exponentially stable. On the other hand, if $1<x_{0}$, then $x\left(t ; t_{0}, x_{0}\right) \rightarrow \infty$ as $t \rightarrow t_{0}+\log \left(x_{0} /\left(x_{0}-1\right)\right)$; that is, in the case that $1<x_{0}$, all solutions are unbounded. Therefore, we can conclude that local theory and global theory 
are completely different concepts. Here, the second question of this paper arises. Will exponential stability guarantee global exponential stability, even if the half-linear differential system (1.1) is nonlinear? We now give the answer to this question.

Theorem 4.1 If the zero solution of (1.1) is uniformly asymptotically stable, then there exist $a \lambda>0$ and $a \beta>0$ such that $t_{0} \in I$ and $\left(x_{0}, y_{0}\right) \in \mathbb{R}^{2}$ imply

$$
\left\|\left(x\left(t ; t_{0}, x_{0}, y_{0}\right), \phi_{p^{*}}\left(y\left(t ; t_{0}, x_{0}, y_{0}\right)\right)\right)\right\|_{p} \leq \beta e^{-\lambda\left(t-t_{0}\right)}\left\|\left(x_{0}, \phi_{p^{*}}\left(y_{0}\right)\right)\right\|_{p}
$$

for all $t \geq t_{0}$, where $\beta>0$ is independent of the size of $\left\|\left(x_{0}, \phi_{p^{*}}\left(y_{0}\right)\right)\right\|_{p}$.

Proof By virtue of Theorem 1.1, it turns out that the uniform asymptotic stability of (1.1) implies exponential stability. Using Lemma 2.3, there exist a $\lambda>0$ and a $\delta(1)>0$ such that $t_{0} \in I$ and $\left\|\left(\xi, \phi_{p^{*}}(\eta)\right)\right\|_{p}<\delta$ imply

$$
\left\|\left(x\left(t ; t_{0}, \xi, \eta\right), \phi_{p^{*}}\left(y\left(t ; t_{0}, \xi, \eta\right)\right)\right)\right\|_{p} \leq e^{-\lambda\left(t-t_{0}\right)}
$$

for all $t \geq t_{0}$.

We choose $\beta=2 / \delta$. Let $t_{0} \in I$ and $\left(x_{0}, y_{0}\right) \in \mathbb{R}^{2}$ be given. We may assume without loss of generality that $\left(x_{0}, y_{0}\right) \neq(0,0)$. Consider the solution $\left(x\left(t ; t_{0}, x_{0}, y_{0}\right), y\left(t ; t_{0}, x_{0}, y_{0}\right)\right)$ of $(1.1)$. For the sake of convenience, we write

$$
(x(t), y(t))=\left(x\left(t ; t_{0}, x_{0}, y_{0}\right), y\left(t ; t_{0}, x_{0}, y_{0}\right)\right), \quad c=\frac{\delta}{2\left\|\left(x_{0}, \phi_{p^{*}}\left(y_{0}\right)\right)\right\|_{p}} .
$$

Hence, we have

$$
\left\|\left(c x_{0}, \phi_{p^{*}}\left(\phi_{p}(c) y_{0}\right)\right)\right\|_{p}=\left\|\left(c x_{0}, c \phi_{p^{*}}\left(y_{0}\right)\right)\right\|_{p}=c\left\|\left(x_{0}, \phi_{p^{*}}\left(y_{0}\right)\right)\right\|_{p}<\delta
$$

Using Lemma 2.4, $\left(c x(t), \phi_{p}(c) y(t)\right)$ is also a solution of (1.1) passing through a point $\left(c x_{0}, \phi_{p}(c) y_{0}\right)$ at $t=t_{0}$. Thus, we get

$$
c\left\|\left(x(t), \phi_{p^{*}}(y(t))\right)\right\|_{p}=\left\|\left(c x(t), \phi_{p^{*}}\left(\phi_{p}(c) y(t)\right)\right)\right\|_{p} \leq e^{-\lambda\left(t-t_{0}\right)}
$$

for all $t \geq t_{0}$, and therefore

$$
\left\|\left(x(t), \phi_{p^{*}}(y(t))\right)\right\|_{p} \leq \frac{2}{\delta} e^{-\lambda\left(t-t_{0}\right)}\left\|\left(x_{0}, \phi_{p^{*}}\left(y_{0}\right)\right)\right\|_{p}=\beta e^{-\lambda\left(t-t_{0}\right)}\left\|\left(x_{0}, \phi_{p^{*}}\left(y_{0}\right)\right)\right\|_{p}
$$

for all $t \geq t_{0}$. This completes the proof of Theorem 4.1.

Theorem 4.1 is a natural generalization of Theorem $\mathrm{D}$ with $n=2$. Actually, in the case that $p=2$, Theorem 4.1 becomes Theorem $\mathrm{D}$ with $n=2$.

Moreover, let us give some definitions. The zero solution of (1.1) is said to be globally uniformly attractive (or uniformly attractive in the large) if for any $\alpha>0$ and any $\varepsilon>0$, there exists a $T(\alpha, \varepsilon)>0$ such that $t_{0} \in I$ and $\left\|\mathbf{x}_{0}\right\|<\alpha$ imply $\left\|\mathbf{x}\left(t ; t_{0}, \mathbf{x}_{0}\right)\right\|<\varepsilon$ for all $t \geq t_{0}+T(\alpha, \varepsilon)$. The solutions of (1.1) are said to be uniformly bounded if, for any $\alpha>0$, there exists a $B(\alpha)>0$ such that $t_{0} \in I$ and $\left\|\mathbf{x}_{0}\right\|<\alpha$ imply $\left\|\mathbf{x}\left(t ; t_{0}, \mathbf{x}_{0}\right)\right\|<B(\alpha)$ for all $t \geq t_{0}$. 
The zero solution of (1.1) is globally uniformly asymptotically stable (or uniformly asymptotically stable in the large) if it is globally uniformly attractive and is uniformly stable and if the solutions of (1.1) are uniformly bounded. For example, we can refer to the books and papers $[7,27-31,33,36,37,47]$ for those definitions. When restricted to the case of the linear system (1.3), the following facts are well known.

Theorem F If the zero solution of (1.3) is uniformly asymptotically stable, then it is globally uniformly asymptotically stable.

We can state a natural generalization of Theorem F with $n=2$ as follows.

Theorem 4.2 If the zero solution of (1.1) is uniformly asymptotically stable, then it is globally uniformly asymptotically stable.

Proof By virtue of Theorem 4.1, if the zero solution of (1.1) is uniformly asymptotically stable, then there exist a $\lambda>0$ and a $\beta>0$ such that $t_{0} \in I$ and $(\xi, \eta) \in \mathbb{R}^{2}$ imply

$$
\left\|\left(x\left(t ; t_{0}, \xi, \eta\right), \phi_{p^{*}}\left(y\left(t ; t_{0}, \xi, \eta\right)\right)\right)\right\|_{p} \leq \beta e^{-\lambda\left(t-t_{0}\right)}\left\|\left(\xi, \phi_{p^{*}}(\eta)\right)\right\|_{p}
$$

for all $t \geq t_{0}$. We have only to show that the zero solution of (1.1) is globally uniformly attractive and the solutions of (1.1) are uniformly bounded.

First we will prove the global uniform attractivity. Let $\varepsilon>0$ and $\alpha>0$ be given. We now consider the solution $\left(x\left(t ; t_{0}, x_{0}, y_{0}\right), y\left(t ; t_{0}, x_{0}, y_{0}\right)\right)$ of $(1.1)$ with $t_{0} \in I$ and $\left\|\left(x_{0}, y_{0}\right)\right\|<\alpha$. For the sake of convenience, we write

$$
(x(t), y(t))=\left(x\left(t ; t_{0}, x_{0}, y_{0}\right), y\left(t ; t_{0}, x_{0}, y_{0}\right)\right), \quad c(\alpha)=\left\|\left(\alpha, \phi_{p^{*}}(\alpha)\right)\right\|_{p} .
$$

We choose a $T(\varepsilon, \alpha)$ such that

$$
T(\varepsilon, \alpha)=\frac{1}{\min \{1, p-1\} \lambda} \log \frac{\sqrt{(\beta c(\alpha))^{2}+(\beta c(\alpha))^{2(p-1)}}}{\varepsilon} .
$$

Since $\left|x_{0}\right|<\alpha$ and $\left|y_{0}\right|<\alpha$, we get

$$
\left\|\left(x_{0}, \phi_{p^{*}}\left(y_{0}\right)\right)\right\|_{p}<c(\alpha) .
$$

We therefore conclude that

$$
|x(t)| \leq\left\|\left(x(t), \phi_{p^{*}}(y(t))\right)\right\|_{p}<\beta c(\alpha) e^{-\lambda\left(t-t_{0}\right)}
$$

and

$$
|y(t)| \leq \phi_{p}\left(\left\|\left(x(t), \phi_{p^{*}}(y(t))\right)\right\|_{p}\right)<\left(\beta c(\alpha) e^{-\lambda\left(t-t_{0}\right)}\right)^{p-1}
$$

for $t \geq t_{0}$. From these inequalities, we see that

$$
\|(x(t), y(t))\|<e^{-\min \{1, p-1\} \lambda\left(t-t_{0}\right)} \sqrt{(\beta c(\alpha))^{2}+(\beta c(\alpha))^{2(p-1)}}
$$


for $t \geq t_{0}$. Hence, we obtain

$$
\|(x(t), y(t))\|<\varepsilon
$$

for $t \geq t_{0}+T$.

We next prove the uniform boundedness. Let $\alpha>0$ be given. As mentioned in the proof of global uniform attractivity, we see that

$$
\|(x(t), y(t))\|<e^{-\min \{1, p-1\} \lambda\left(t-t_{0}\right)} \sqrt{(\beta c(\alpha))^{2}+(\beta c(\alpha))^{2(p-1)}}
$$

for $t \geq t_{0}$ and $\left\|\left(x_{0}, y_{0}\right)\right\|<\alpha$, where

$$
(x(t), y(t))=\left(x\left(t ; t_{0}, x_{0}, y_{0}\right), y\left(t ; t_{0}, x_{0}, y_{0}\right)\right), \quad c(\alpha)=\left\|\left(\alpha, \phi_{p^{*}}(\alpha)\right)\right\|_{p} .
$$

We choose a $B(\alpha)>0$ such that

$$
B(\alpha)=\sqrt{(\beta c(\alpha))^{2}+(\beta c(\alpha))^{2(p-1)}} .
$$

Hence, we obtain

$$
\left\|\left(x\left(t ; t_{0}, x_{0}, y_{0}\right), y\left(t ; t_{0}, x_{0}, y_{0}\right)\right)\right\|<B(\alpha)
$$

for $t \geq t_{0}$. This completes the proof of Theorem 4.2 .

The claim of Theorem 4.2 contributes to clear that uniform asymptotic stability and global uniform asymptotic stability are completely same concept for the half-linear differential system (1.1). Moreover, we can conclude that uniform asymptotic stability, exponential stability and global exponential stability are equivalent for the half-linear differential system (1.1) from the results of Theorems 1.1 and 4.1 .

\section{Converse theorems on exponential stability}

In this section, for comparison with Theorems $\mathrm{B}, \mathrm{C}$ and $\mathrm{E}$, we will discuss the converse theorems for half-linear system (1.1). Recall that the right-hand side of (1.1) does not satisfy Lipschitz condition at the origin. For this reason, unfortunately, Theorems B, C and E cannot apply to (1.1). First, let us consider the existence of a Lyapunov function estimated by the form $\|\mathbf{x}\|_{p}^{p}$. For this purpose, we give a lemma as follows.

Lemma 5.1 If $(x(t), y(t))$ is a solution of (1.1) passing through a point $\left(x_{0}, y_{0}\right) \in \mathbb{R}^{2}$ at $t=$ $t_{0} \in I$, then the following inequality holds:

$$
\left\|\left(x(t), \phi_{p^{*}}(y(t))\right)\right\|_{p}^{p} \geq\left\|\left(x_{0}, \phi_{p^{*}}\left(y_{0}\right)\right)\right\|_{p}^{p} \exp \left(\int_{t_{0}}^{t} \psi(s) d s\right)
$$

for $t \geq t_{0}$, where the continuous function $\psi(t)$ defined by

$$
\psi(t)=\min \left\{p a_{11}(t)-\left|(p-1) a_{12}(t)+a_{21}(t)\right|, p^{*} a_{22}(t)-\left|a_{12}(t)+\left(p^{*}-1\right) a_{21}(t)\right|\right\} .
$$


Proof Define

$$
w(t)=\left\|\left(x(t), \phi_{p^{*}}(y(t))\right)\right\|_{p}^{p}=|x(t)|^{p}+|y(t)|^{p^{*}}
$$

for $t \geq t_{0}$. From the right-hand side of (1.1), we have

$$
\begin{aligned}
w^{\prime}(t)= & p a_{11}(t)|x(t)|^{p}+p^{*} a_{22}(t)|y(t)|^{p^{*}} \\
& +\left(p a_{12}(t)+p^{*} a_{21}(t)\right) \phi_{p}(x(t)) \phi_{p^{*}}(y(t))
\end{aligned}
$$

for $t \geq t_{0}$. Using the classical Young inequality, we obtain

$$
\begin{aligned}
w^{\prime}(t) \geq & p a_{11}(t)|x(t)|^{p}+p^{*} a_{22}(t)|y(t)|^{p^{*}}-\left|p a_{12}(t)+p^{*} a_{21}(t)\right||x(t)|^{p-1}|y(t)|^{p^{*}-1} \\
\geq & p a_{11}(t)|x(t)|^{p}+p^{*} a_{22}(t)|y(t)|^{p^{*}} \\
& \quad-\left|p a_{12}(t)+p^{*} a_{21}(t)\right|\left(\frac{|x(t)|^{(p-1) p^{*}}}{p^{*}}+\frac{|y(t)|^{\left(p^{*}-1\right) p}}{p}\right) \\
= & \left(p a_{11}(t)-\left|(p-1) a_{12}(t)+a_{21}(t)\right|\right)|x(t)|^{p} \\
& +\left(p^{*} a_{22}(t)-\left|a_{12}(t)+\left(p^{*}-1\right) a_{21}(t)\right|\right)|y(t)|^{p^{*}} \\
\geq & \psi(t) w(t)
\end{aligned}
$$

for $t \geq t_{0}$. Therefore, we get

$$
\left(\exp \left(-\int_{t_{0}}^{t} \psi(s) d s\right) w(t)\right)^{\prime} \geq 0
$$

for $t \geq t_{0}$. Integrate this inequality from $t_{0}$ to $t$ to obtain

$$
w(t) \geq w\left(t_{0}\right) \exp \left(\int_{t_{0}}^{t} \psi(s) d s\right)=\left\|\left(x_{0}, \phi_{p^{*}}\left(y_{0}\right)\right)\right\|_{p}^{p} \exp \left(\int_{t_{0}}^{t} \psi(s) d s\right)
$$

for $t \geq t_{0}$.

The first converse theorem of this section is as follows. We can prove this theorem without requiring the uniqueness of solutions of (1.1) for the initial value problem.

Theorem 5.2 Suppose that all coefficients of (1.1) are bounded on I and that there exist a $\lambda>0$ and $a \beta>0$ such that

$$
\left\|\left(x\left(t ; t_{0}, x_{0}, y_{0}\right), \phi_{p^{*}}\left(y\left(t ; t_{0}, x_{0}, y_{0}\right)\right)\right)\right\|_{p} \leq \beta e^{-\lambda\left(t-t_{0}\right)}\left\|\left(x_{0}, \phi_{p^{*}}\left(y_{0}\right)\right)\right\|_{p}
$$

for all $t \geq t_{0} \geq 0$, where $\left(x\left(t ; t_{0}, x_{0}, y_{0}\right), y\left(t ; t_{0}, x_{0}, y_{0}\right)\right)$ is a solution of $(1.1)$. Then there exist three positive constants $\beta_{1}, \beta_{2}, \beta_{3}$ and a Lyapunov function $V(t, x, y)$ defined on $I \times \mathbb{R}^{2}$ which satisfies the following conditions:

(i) $\beta_{1}\left\|\left(x, \phi_{p^{*}}(y)\right)\right\|_{p}^{p} \leq V(t, x, y) \leq \beta_{2}\left\|\left(x, \phi_{p^{*}}(y)\right)\right\|_{p}^{p}$;

(ii) $\dot{V}_{(1.1)}(t, x, y) \leq-\beta_{3}\left\|\left(x, \phi_{p^{*}}(y)\right)\right\|_{p}^{p}$. 
Proof From the assumption, all solutions $(x(s ; t, x, y), y(s ; t, x, y))$ of (1.1) passing through a point $(x, y) \in \mathbb{R}^{2}$ at $t \in I$ satisfy

$$
\left\|\left(x(s ; t, x, y), \phi_{p^{*}}(y(s ; t, x, y))\right)\right\|_{p} \leq \beta\left\|\left(x, \phi_{p^{*}}(y)\right)\right\|_{p}
$$

for $s \geq t$. Therefore, we can consider the function

$$
\bar{v}(s ; t, x, y)=\sup \left\|\left(x(s ; t, x, y), \phi_{p^{*}}(y(s ; t, x, y))\right)\right\|_{p}
$$

for $s \geq t$. Note that if we suppose the uniqueness of solutions of (1.1) for the initial value problem, then $\bar{v}(s ; t, x, y)=\left\|\left(x(s ; t, x, y), \phi_{p^{*}}(y(s ; t, x, y))\right)\right\|_{p}$ holds for $s \geq t$. However, we can prove this theorem without requiring the uniqueness of solutions. Let $V(t, x, y)$ be defined by

$$
V(t, x, y)=\int_{t}^{t+T} \bar{v}^{p}(s ; t, x, y) d s
$$

where $T=(1 / \lambda) \log (\beta \sqrt[p]{2})$ is a constant. From the assumption, we obtain the following estimate:

$$
\begin{aligned}
V(t, x, y) & \leq \int_{t}^{t+T} e^{-p \lambda(s-t)} d s\left(\beta\left\|\left(x, \phi_{p^{*}}(y)\right)\right\|_{p}\right)^{p} \\
& =\frac{\beta^{p}\left(1-e^{-p \lambda T}\right)}{p \lambda}\left\|\left(x, \phi_{p^{*}}(y)\right)\right\|_{p}^{p}=\beta_{2}\left\|\left(x, \phi_{p^{*}}(y)\right)\right\|_{p}^{p} .
\end{aligned}
$$

We will show that $\beta_{1}\left\|\left(x, \phi_{p^{*}}(y)\right)\right\|_{p}^{p} \leq V(t, x, y)$. Since all coefficients of (1.1) are bounded on $I$, there exists an $L>0$ such that $|\psi(s)| \leq L$ for all $s \in I$, where $\psi$ is the continuous function given in Lemma 5.1. From Lemma 5.1, we have

$$
\bar{v}^{p}(s ; t, x, y) \geq \exp \left(\int_{t}^{s} \psi(\tau) d \tau\right)\left\|\left(x, \phi_{p^{*}}(y)\right)\right\|_{p}^{p} \geq e^{-L(s-t)}\left\|\left(x, \phi_{p^{*}}(y)\right)\right\|_{p}^{p}
$$

for $s \geq t$. Thus, we get

$$
\begin{aligned}
V(t, x, y) & \geq \int_{t}^{t+T} e^{-L(s-t)} d s\left\|\left(x, \phi_{p^{*}}(y)\right)\right\|_{p}^{p} \\
& =\frac{1-e^{-L T}}{L}\left\|\left(x, \phi_{p^{*}}(y)\right)\right\|_{p}^{p}=\beta_{1}\left\|\left(x, \phi_{p^{*}}(y)\right)\right\|_{p}^{p} .
\end{aligned}
$$

Therefore, condition (i) is satisfied.

We next prove the condition (ii). Let $h>0$ and

$$
(x(s), y(s))=(x(s ; t, x, y), y(s ; t, x, y)) \quad \text { for } s \geq t .
$$

From the definition of $\bar{v}$, we see that

$$
\bar{v}(s ; u, x(u), y(u)) \leq \bar{v}(s ; t, x, y)
$$


for $t \leq u \leq s$. Then we get

$$
\begin{aligned}
V(t+h, x(t+h), y(t+h))= & \int_{t+h}^{t+h+T} \bar{v}^{p}(s ; t+h, x(t+h), y(t+h)) d s \\
\leq & \int_{t+h}^{t+h+T} \bar{v}^{p}(s ; t, x, y) d s \\
= & V(t, x, y)+\int_{t+T}^{t+h+T} \bar{v}^{p}(s ; t, x, y) d s-\int_{t}^{t+h} \bar{v}^{p}(s ; t, x, y) d s \\
\leq & V(t, x, y)+\int_{t+T}^{t+h+T} \beta^{p} e^{-p \lambda(s-t)}\left\|\left(x, \phi_{p^{*}}(y)\right)\right\|_{p}^{p} d s \\
& -\int_{t}^{t+h} e^{-L(s-t)}\left\|\left(x, \phi_{p^{*}}(y)\right)\right\|_{p}^{p} d s \\
= & V(t, x, y) \\
& +\left(\frac{\beta^{p} e^{-p \lambda T}\left(1-e^{-p \lambda h}\right)}{p \lambda}-\frac{1-e^{-L h}}{L}\right)\left\|\left(x, \phi_{p^{*}}(y)\right)\right\|_{p}^{p}
\end{aligned}
$$

from the assumption and Lemma 5.1. Therefore, we can estimate that

$$
\begin{aligned}
& \frac{1}{h}(V(t+h, x(t+h), y(t+h))-V(t, x, y)) \\
& \quad \leq\left(\frac{\beta^{p} e^{-p \lambda T}\left(1-e^{-p \lambda h}\right)}{p \lambda h}-\frac{1-e^{-L h}}{L h}\right)\left\|\left(x, \phi_{p^{*}}(y)\right)\right\|_{p}^{p} .
\end{aligned}
$$

From this inequality and

$$
\lim _{h \rightarrow 0}\left(\frac{\beta^{p} e^{-p \lambda T}\left(1-e^{-p \lambda h}\right)}{p \lambda h}-\frac{1-e^{-L h}}{L h}\right)=\beta^{p} e^{-p \lambda T}-1=-\frac{1}{2},
$$

we obtain

$$
\dot{V}_{(1.1)}(t, x, y) \leq-\frac{1}{2}\left\|\left(x, \phi_{p^{*}}(y)\right)\right\|_{p}^{p}=-\beta_{3}\left\|\left(x, \phi_{p^{*}}(y)\right)\right\|_{p}^{p} .
$$

This completes the proof of Theorem 5.2.

Note that three positive constants $\beta_{1}, \beta_{2}, \beta_{3}$ in Theorem 5.2 are independent of the size of $\left\|\left(x_{0}, \phi_{p^{*}}\left(y_{0}\right)\right)\right\|_{p}$. The second converse theorem is as follows.

Theorem 5.3 Suppose that there exist $a \lambda>0$ and $a \beta>0$ such that

$$
\left\|\left(x\left(t ; t_{0}, x_{0}, y_{0}\right), \phi_{p^{*}}\left(y\left(t ; t_{0}, x_{0}, y_{0}\right)\right)\right)\right\|_{p} \leq \beta e^{-\lambda\left(t-t_{0}\right)}\left\|\left(x_{0}, \phi_{p^{*}}\left(y_{0}\right)\right)\right\|_{p}
$$

for all $t \geq t_{0} \geq 0$, where $\left(x\left(t ; t_{0}, x_{0}, y_{0}\right), y\left(t ; t_{0}, x_{0}, y_{0}\right)\right)$ is a solution of $(1.1)$. Then there exists a Lyapunov function $V(t, x, y)$ defined on $I \times \mathbb{R}^{2}$ which satisfies the following conditions:

(i) $\left\|\left(x, \phi_{p^{*}}(y)\right)\right\|_{p} \leq V(t, x, y) \leq \beta\left\|\left(x, \phi_{p^{*}}(y)\right)\right\|_{p}$;

(ii) $\dot{V}_{(1.1)}(t, x, y) \leq-\lambda V(t, x, y)$.

Proof Let $V(t, x, y)$ be defined by

$$
V(t, x, y)=\sup _{\sigma \geq 0} \bar{v}(t+\sigma ; t, x, y) e^{\lambda \sigma},
$$


where $\bar{v}$ is the function given in the proof of Theorem 5.2. It is clear that $\left\|\left(x, \phi_{p^{*}}(y)\right)\right\|_{p} \leq$ $V(t, x, y)$. On the other hand, we can easy to see that

$$
V(t, x, y) \leq \sup _{\sigma \geq 0} \beta e^{-\lambda \sigma}\left\|\left(x, \phi_{p^{*}}(y)\right)\right\|_{p} e^{\lambda \sigma}=\beta\left\|\left(x, \phi_{p^{*}}(y)\right)\right\|_{p}
$$

by the assumption.

We next prove the condition (ii). Let $h>0$ and

$$
(x(s), y(s))=(x(s ; t, x, y), y(s ; t, x, y)) \quad \text { for } s \geq t .
$$

Then we get

$$
\begin{aligned}
V(t+h, x(t+h), y(t+h)) & =\sup _{\sigma \geq 0} \bar{v}(t+h+\sigma ; t+h, x(t+h), y(t+h)) e^{\lambda \sigma} \\
& =\sup _{\tau \geq h} \bar{v}(t+\tau ; t+h, x(t+h), y(t+h)) e^{\lambda \tau} e^{-\lambda h} \\
& \leq \sup _{\tau \geq h} \bar{v}(t+\tau ; t, x, y) e^{\lambda \tau} e^{-\lambda h} \\
& \leq \sup _{\tau \geq 0} \bar{v}(t+\tau ; t, x, y) e^{\lambda \tau} e^{-\lambda h}=V(t, x, y) e^{-\lambda h}
\end{aligned}
$$

from $\bar{v}(s ; u, x(u), y(u)) \leq \bar{v}(s ; t, x, y)$ for $t \leq u \leq s$. Therefore, we can estimate that

$$
\frac{V(t+h, x(t+h), y(t+h))-V(t, x, y)}{h} \leq \frac{e^{-\lambda h}-1}{h} V(t, x, y) .
$$

From this inequality and

$$
\lim _{h \rightarrow 0} \frac{e^{-\lambda h}-1}{h}=-\lambda,
$$

we obtain

$$
\dot{V}_{(1.1)}(t, x, y) \leq-\lambda V(t, x, y) .
$$

This completes the proof of Theorem 5.3.

\section{Competing interests}

The authors declare that they have no competing interests.

Authors' contributions

Both authors contributed equally to the writing of this paper. Both authors read and approved the final manuscript.

\section{Acknowledgements}

The first author's work was supported in part by Grant-in-Aid for Young Scientists (B), No. 23740115 from the Japan Society for the Promotion of Science (JSPS). The authors thank the referee for useful and valuable comments.

Received: 30 January 2015 Accepted: 4 May 2015 Published online: 16 May 2015

\section{References}

1. Došlý, O: Half-linear differential equations. In: Handbook of Differential Equations, pp. 161-357. Elsevier, Amsterdam (2004)

2. Došlý, O, Rehák, P: Half-Linear Differential Equations. North-Holland Mathematics Studies, vol. 202. Elsevier, Amsterdam (2005) 
3. Elbert, Á: Asymptotic behaviour of autonomous half-linear differential systems on the plane. Studia Sci. Math. Hung $19(2-4), 447-464(1984)$

4. Mirzov, JD: On some analogs of Sturm's and Kneser's theorems for nonlinear systems. J. Math. Anal. Appl. 53(2), 418-425 (1976)

5. Mirzov, JD: Principal and nonprincipal solutions of a nonlinear system. Tbil. Gos. Univ. Inst. Prikl. Mat. Tr. 31, 100-117 (1988) (Russian)

6. Mirzov, JD: Asymptotic Properties of Solutions of Systems of Nonlinear Nonautonomous Ordinary Differential Equations. Folia Facultatis Scientiarium Naturalium Universitatis Masarykianae Brunensis. Mathematica, vol. 14. Masaryk University, Brno (2004)

7. Onitsuka, M, Sugie, J: Uniform global asymptotic stability for half-linear differential systems with time-varying coefficients. Proc. R. Soc. Edinb., Sect. A 141(5), 1083-1101 (2011)

8. Sugie, J, Hata, S, Onitsuka, M: Global attractivity for half-linear differential systems with periodic coefficients. J. Math. Anal. Appl. 371(1), 95-112 (2010)

9. Sugie, J, Onitsuka, M: Global asymptotic stability for half-linear differential systems with coefficients of indefinite sign. Arch. Math. 44(4), 317-334 (2008)

10. Agarwal, RP, Grace, SR, O'Regan, D: Oscillation Theory for Second Order Linear, Half-Linear, Superlinear and Sublinear Dynamic Equations. Kluwer Academic, Dordrecht (2002)

11. Elbert, Á: A half-linear second order differential equation. In: Qualitative Theory of Differential Equations (Szeged, 1979), vols. I, II. Colloq. Math. Soc. János Bolyai, vol. 30, pp. 153-180. North-Holland, Amsterdam (1981)

12. Hasil, $\mathrm{P}$, Mařik, R, Veselý, M: Conditional oscillation of half-linear differential equations with coefficients having mean values. Abstr. Appl. Anal. 2014, Article ID 258159 (2014)

13. Hatvani, L: On stability properties of solutions of second order differential equations. Proceedings of the 6 th Colloquium on the Qualitative Theory of Differential Equations (Szeged, 1999). Electron. J. Qual. Theory Differ. Equ 2000, 11 (2000)

14. Pašić, M, Wong, JSW: Rectifiable oscillations in second-order half-linear differential equations. Ann. Mat. Pura Appl. (4) 188(3), 517-541 (2009)

15. Řehák, P: De Haan type increasing solutions of half-linear differential equations. J. Math. Anal. Appl. 412(1), 236-243 (2014)

16. Sugie, J, Matsumura, K: A nonoscillation theorem for half-linear differential equations with periodic coefficients. Appl. Math. Comput. 199(2), 447-455 (2008)

17. Sugie, J, Onitsuka, M: Global asymptotic stability for damped half-linear differential equations. Acta Sci. Math. 73(3-4), 613-636 (2007)

18. Sugie, J, Onitsuka, M: Integral conditions on the uniform asymptotic stability for two-dimensional linear systems with time-varying coefficients. Proc. Am. Math. Soc. 138(7), 2493-2503 (2010)

19. Sugie, J, Onitsuka, M: Growth conditions for uniform asymptotic stability of damped oscillators. Nonlinear Anal. 98 , 83-103 (2014)

20. Agarwal, RP, Bohner, M, Li, T: Oscillatory behavior of second-order half-linear damped dynamic equations. Appl. Math. Comput. 254, 408-418 (2015)

21. Došlý, O, Fišnarová, S: Linearized Riccati technique and (non-)oscillation criteria for half-linear difference equations. Adv. Differ. Equ. 2008, Article ID 438130 (2008)

22. Došlý, O, Yamaoka, N: Oscillation constants for second-order ordinary differential equations related to elliptic equations with $p$-Laplacian. Nonlinear Anal. 113, 115-136 (2015)

23. Fišnarová, S: Oscillatory properties of half-linear difference equations: two-term perturbations. Adv. Differ. Equ. 2012, $101(2012)$

24. Hasil, P, Mařik, R, Veselý, M: Oscillation and nonoscillation of asymptotically almost periodic half-linear difference equations. Abstr. Appl. Anal. 2013, Article ID 432936 (2014)

25. Halanay, A: Differential Equations: Stability, Oscillations, Time Lags. Academic Press, New York (1966)

26. Lefschetz, S: Differential Equations: Geometric Theory, 2nd edn. Pure and Applied Mathematics, vol. 6. Interscience, New York (1963)

27. Sansone, G, Conti, R: Non-linear Differential Equations. International Series of Monographs in Pure and Applied Mathematics, vol. 67. Macmillan Co., New York (1964). Revised ed. Translated from the Italian by Ainsley H Diamond

28. Yoshizawa, T: Stability Theory by Liapunov's Second Method. The Mathematical Society of Japan, Tokyo (1966)

29. Yoshizawa, T: Stability Theory and the Existence of Periodic Solutions and Almost Periodic Solutions. Applied Mathematical Sciences, vol. 14. Springer, New York (1975)

30. Bacciotti, A, Rosier, L: Liapunov Functions and Stability in Control Theory, 2nd edn. Communications and Control Engineering Series. Springer, Berlin (2005)

31. Hahn, W: Stability of Motion. Die Grundlehren der Mathematischen Wissenschaften, vol. 138. Springer, New York (1967). Translated from the German manuscript by Arne P Baartz

32. Hale, JK: Ordinary Differential Equations. Pure and Applied Mathematics, vol. 11. Wiley-Interscience, New York (1969)

33. Loría, A, Panteley, E: Stability, told by its developers. In: Advanced Topics in Control Systems Theory. Lecture Notes in Control and Inform. Sci., vol. 328, pp. 199-258. Springer, London (2006)

34. Malisoff, M, Mazenc, F: Constructions of Strict Lyapunov Functions. Communications and Control Engineering Series. Springer, London (2009)

35. Michel, AN, Hou, L, Liu, D: Stability of Dynamical Systems: Continuous, Discontinuous, and Discrete Systems. Systems and Control: Foundations and Applications. Birkhäuser Boston, Boston (2008)

36. Rouche, N, Habets, P, Laloy, M: Stability Theory by Liapunov's Direct Method. Applied Mathematical Sciences, vol. 22. Springer, New York (1977)

37. Harris, CJ, Miles, JF: Stability of Linear Systems. Mathematics in Science and Engineering, vol. 153. Academic Press, New York (1980)

38. Logemann, H, Ryan, EP: Ordinary Differential Equations: Analysis, Qualitative Theory and Control. Springer Undergraduate Mathematics Series. Springer, London (2014)

39. Barreira, L, Pesin, Y: Introduction to Smooth Ergodic Theory. Graduate Studies in Mathematics, vol. 148. Am. Math Soc., Providence (2013) 
40. Burton, TA: Stability and Periodic Solutions of Ordinary and Functional Differential Equations. Mathematics in Science and Engineering, vol. 178. Academic Press, San Diego (1985)

41. Cesari, L: Asymptotic Behavior and Stability Problems in Ordinary Differential Equations, 3rd edn. Ergebnisse der Mathematik und ihrer Grenzgebiete, vol. 16. Springer, New York (1971)

42. Coppel, WA: Stability and Asymptotic Behavior of Differential Equations. Heath, Boston (1965)

43. Halanay, A, Răsvan, V: Applications of Liapunov Methods in Stability. Mathematics and Its Applications, vol. 245. Kluwer Academic, Dordrecht (1993)

44. Hatvani, L: On the uniform attractivity of solutions of ordinary differential equations by two Lyapunov functions. Proc. Jpn. Acad., Ser. A, Math. Sci. 67(5), 162-167 (1991)

45. Ignatyev, AO: Stability of a linear oscillator with variable parameters. Electron. J. Differ. Equ. 1997, 17 (1997)

46. LaSalle, JP, Lefschetz, S: Stability by Liapunov's Direct Method with Applications. Mathematics in Science and Engineering, vol. 4. Academic Press, New York (1961)

47. Malkin, IG: On the question of the reciprocal of Lyapunov's theorem on asymptotic stability. Prikl. Mat. Meh. 18, 129-138 (1954)

48. Massera, JL: Contributions to stability theory. Ann. Math. (2) 64, 182-206 (1956)

49. Onitsuka, M: Non-uniform asymptotic stability for the damped linear oscillator. Nonlinear Anal. 72(3-4), 1266-1274 (2010)

50. Onitsuka, M: Uniform asymptotic stability for damped linear oscillators with variable parameters. Appl. Math. Comput. 218(4), 1436-1442 (2011)

51. Strauss, A, Yorke, JA: Perturbing uniform asymptotically stable nonlinear systems. J. Differ. Equ. 6, $452-483$ (1969)

52. Sugie, J, Ogami, Y, Onitsuka, M: Asymptotic stability for quasi-linear systems whose linear approximation is not assumed to be uniformly attractive. Ann. Mat. Pura Appl. (4) 190(3), 409-425 (2011)

53. Yang, T: Impulsive Control Theory. Lecture Notes in Control and Information Sciences, vol. 272. Springer, Berlin (2001)

54. Agarwal, RP, O'Regan, D: An Introduction to Ordinary Differential Equations. Springer, New York (2008)

55. Mahmudov, E: Single Variable Differential and Integral Calculus: Mathematical Analysis. Atlantis Press, Paris (2013)

\section{Submit your manuscript to a SpringerOpen ${ }^{\circ}$ journal and benefit from:}

- Convenient online submission

- Rigorous peer review

Immediate publication on acceptance

Open access: articles freely available online

- High visibility within the field

- Retaining the copyright to your article 